\title{
Beat the giant: case of a giant prolactinoma during pregnancy on cabergoline
}

\author{
Wael M Almistehi1 and Mussa H Almalki1,2 \\ 1Obesity, Endocrine, and Metabolism Center, King Fahad Medical City, Riyadh, Saudi Arabia and 2King Fahad Medical \\ City, College of Medicine, King Saud bin Abdulaziz University for Health Sciences, Riyadh, Saudi Arabia
}

Correspondence should be addressed to W M Almistehi Email

wael.almistehi@gmail.com

\section{Summary}

Giant prolactinomas are a rare entity; during pregnancy, the risk for complications associated with these tumors is higher. Here, we report a case of a young woman who had an invasive, giant prolactinoma post resection with residual disease who became pregnant. This patient was treated with cabergoline to prevent tumor expansion in pregnancy, resulting in the uneventful delivery of a healthy baby boy.

\section{Learning points:}

- Giant prolactinoma can cause both diagnostic and therapeutic challenges given their atypical presentation.

- Accurate diagnosis is paramount to avoid unnecessary surgical intervention or pituitary irradiation.

- This case demonstrates the effectiveness and safety of CAB therapy during pregnancy.

\section{Background}

Pituitary tumors account for $10-15 \%$ of all intracranial tumors. Prolactinomas are the most frequently occurring pituitary tumors with a prevalence of 3.5-5 per 10000 inhabitants, according to recent epidemiological studies $(1,2,3)$. Prolactinomas are typically microadenomas and they occur more often in women (3), while macroadenomas are usually found in men $(4,5)$. Giant prolactinomas are a subset of macroadenomas, defined as tumors $>4 \mathrm{~cm}$ in diameter; they are associated with prolactin (PRL) levels $>1000 \mathrm{ng} / \mathrm{mL}$ and typically result in clinical symptoms such as hyperprolactinemia or they present with mass effects (5). Although prolactinomas are benign, giant macroprolactinomas are often locally aggressive, invading surrounding structures $(6,7)$.

Dopamine agonists (DA) are the first-line treatment for prolactinomas, as these agents can rapidly alleviate neurological symptoms. Further, they are highly effective at normalizing PRL levels, and they induce significant shrinkage of tumor volume in both men and women $(8,9,10)$. Surgery is usually reserved for patients who are intolerant to - or exhibit an inadequate response to - DA; it is also recommended for patients with invasive macroadenomas, as the procedure can be used for tumor debulking and to alleviate neurological deficits (11). Radiotherapy is only used in cases of surgical failure or resistance to DA (12).

During pregnancy, the pituitary gland undergoes lactotroph hyperplasia due to a progressive increase in serum estrogen levels, possibly resulting in a substantial increase in tumor volume (4). The risk of tumor growth is minimal in microadenomas $(2.7 \%)$, being higher in macroadenomas $(15 \%-35 \%)(1,3)$. It is recommended that patients discontinue DA therapy once pregnancy is confirmed, usually 1-2 weeks after a missed period, limiting potential fetal exposure to only 3-4 weeks (13).

Although prolactinomas are amenable to medical therapy with DA during pregnancy, it is clear that taking bromocriptine and cabergoline $(\mathrm{CAB})$ until conception is safe; however, there is scant evidence to support the idea that continuing the use of bromocriptine or $\mathrm{CAB}$ is 
safe during pregnancy. The Endocrine Society guidelines recommend discontinuing DAs as soon as pregnancy is confirmed, except for women harboring invasive macroprolactinomas or in patients with evidence of tumor growth and visual and/or headache complaints (14).

Patients should be monitored and evaluated every trimester or more frequently for those with larger tumors, for changes in symptoms such as headaches and visual field defects (14). Here, we describe the case of an invasive, giant prolactinoma, which was misdiagnosed due to a falsely low PRL concentration. In the case described here, the patient became pregnant and was successfully treated with $\mathrm{CAB}$ throughout her pregnancy.

\section{Case report}

A 29-year-old Saudi female - married, and a mother of two children - presented with nasal obstruction, secondary amenorrhea, and visual disturbance. She initially saw her ear, nose and throat surgeon and her initial investigations revealed an elevated PRL level of $142.5 \mathrm{ng} / \mathrm{mL}$ (normal range: $4.15-23.2 \mathrm{ng} / \mathrm{mL}$ ) using the Cobas assay by Roche (Hoffman-La Roche, Basel, Switzerland). MRI showed a sellar mass measuring $6.2 \times 4.22 \mathrm{~cm}$, invading the cavernous sinuses and skull base bones and extending to the nasal cavity, paranasal sinuses and pterygopalatine fosse (Fig. 1A and B), increasing the possibility of the presence of a non-functioning pituitary adenoma. Her visual field examination was normal. She underwent transsphenoidal surgery in November 2013.

The postoperative period was uneventful without any complications. Imaging by CT scans of the brain and MRI scans of the pituitary post-surgery showed a residual pituitary tumor, with removal of $60 \%$ of the para-sellar lesion (Fig. 1C and D). The other relevant pituitary hormones were very much within the normal limits, apart from hypothyroidism. The patient was prescribed levothyroxine (75 mg daily) and discharged home in good condition.

The resected tumor tissue showed strong and positive immunostaining for PRL, but it was negative for the other pituitary hormones. After consultation with the endocrinologist, her PRL levels with dilution revealed an increased PRL concentration (3380.95 ng/mL), confirming the diagnosis of an invasive giant prolactinoma.

After surgery, given her positive immunostaining for PRL and high PRL levels after dilution, as well as the presence of gross tumor remnants, treatment was attempted with cabergoline (CAB) at a dose of $0.25 \mathrm{mg}$ twice a week, which improved, stabilized and controlled
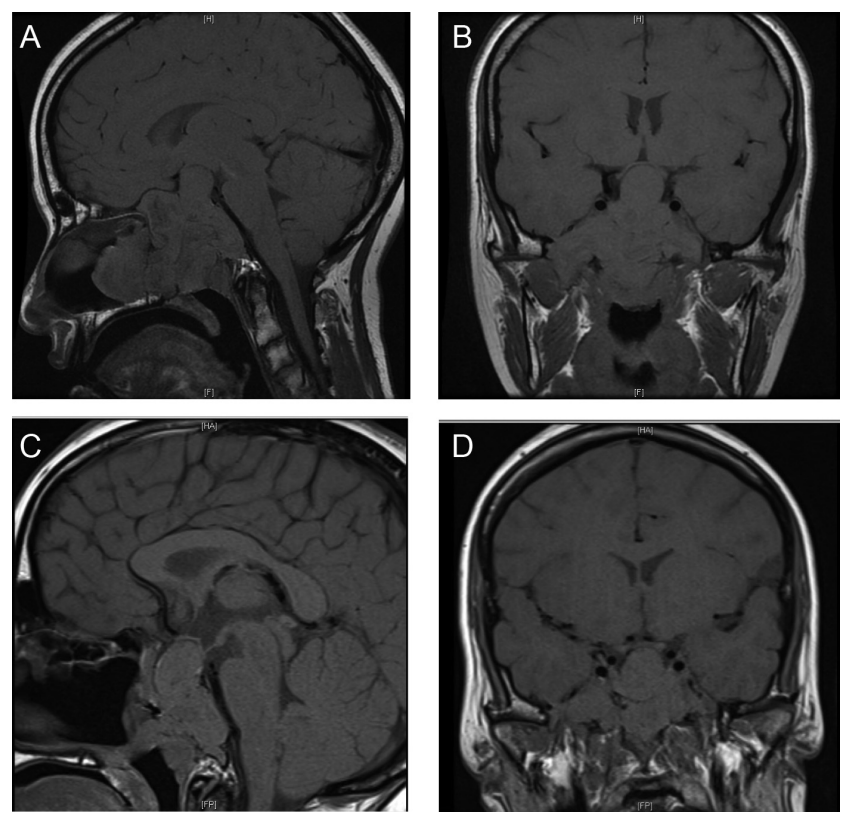

Figure 1

(A and B) Preoperative MRI scan of the pituitary showing a large sellar/ suprasellar mass, which invaded the bilateral cavernous sinuses and skull base bones and extended to the nasal cavity, paranasal sinuses and pterygopalatine fosse. (C and D) Postoperative MRI scan of the pituitary showing a residual lesion that appeared to be stable in size and signal intensity at the sellar and suprasellar component, invading the skull base and extending to the nasopharynx. The optic chiasm and optic nerves appear unremarkable; the bilateral internal carotid arteries are again noted to be encircled by the tumor and are still patent.

the disease based on biochemical and radiological findings, and the patient achieved regular menses. The patient asked about the possibility of pregnancy and had been advised to avoid pregnancy due to the substantial risk of tumor growth during pregnancy. The patient's medication was maintained at the same dose, as she showed good biochemical and radiological response to CAB.

In 2016, the patient attended our clinic reporting a 6-week pregnancy that she wanted to continue. Given the tumor characteristics, she was advised to continue $\mathrm{CAB}$ at a dose of $0.25 \mathrm{mg}$ twice weekly, followed by monthly clinical visits; monitoring appointments of her high-risk pregnancy was scheduled.

Given the potential risk of tumor enlargement, an MRI pituitary was performed without gadolinium at 26 weeks of gestation which showed a slight increase in adenoma size (Fig. 2A and B), but no headaches or visual disturbances were reported. Ophthalmological and visual field examinations were completely normal. The patient was advised to increase the $\mathrm{CAB}$ dose to $0.5 \mathrm{mg}$ twice per week to ensure a further reduction in tumor size, but she declined to do so. 

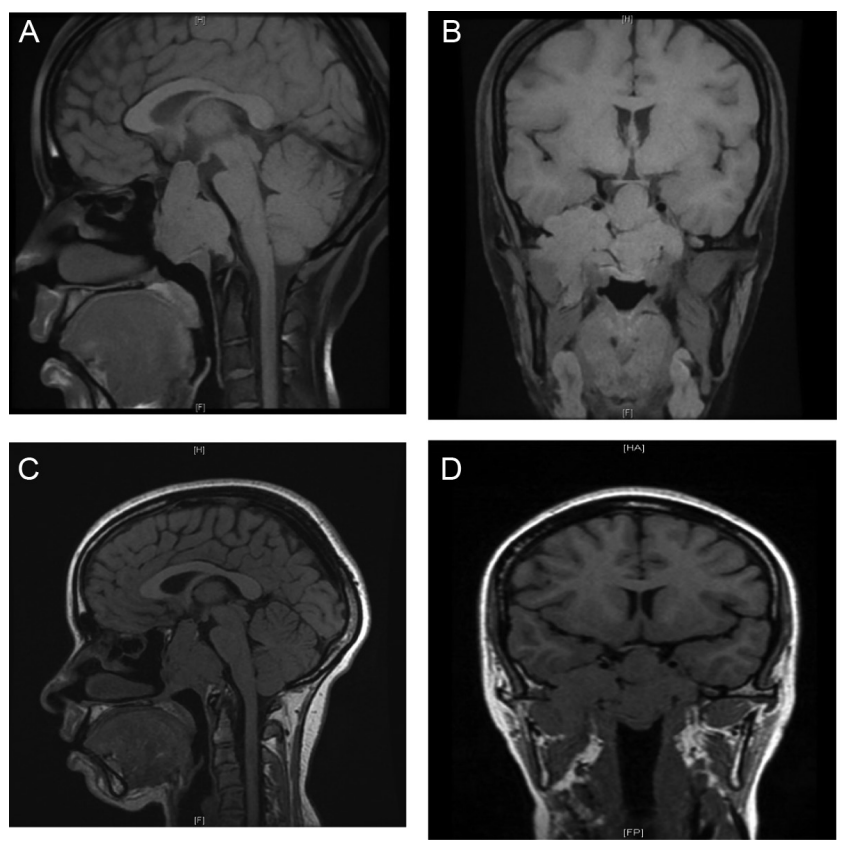

Figure 2

(A and B) Intrapartum (26 weeks' gestation) MRI scan of the pituitary showing a minimal increase in the size of the macroadenoma in the anterior-posterior dimension. The supratentorial component of the tumor just reaches the margin of the optic chiasm. The optic chiasm is thin and moderately atrophied with a mild T2 hyperintense signal abnormality, unchanged as compared with prior studies. (C and D) Postpartum MRI scan of the pituitary showed interval tumor progression since the previous MRI scan. The tumor currently shows more progression extracranially, affecting the intratemporal region and eroding the floor of the right-middle cranial fossa. It subsequently developed into a dural-based enhancing lesion that was noted in continuity with the previous tumor, causing compression and displacement to the right temporal pole. Furthermore, interval progression was noted in the size of the tumor at the roof of the nasopharynx.

The patient remained symptom-free during gestation; she had normal perimetry tests every 3 months until she vaginally delivered a normal, full-term, healthy baby boy. The baby weighed $2.3 \mathrm{~kg}$, and the baby had normal postnatal development.

The postpartum period was uneventful, and $\mathrm{CAB}$ was continued at a dose of $0.25 \mathrm{mg}$ twice per week. The patient's PRL levels, as illustrated in Table 1, remained within the normal range $(16.6 \mathrm{ng} / \mathrm{mL})$, and an MRI scan showed tumor enlargement (Fig. 2C and D), although the patient reported no symptoms. To decrease the morbidity associated with tumor growth, the $\mathrm{CAB}$ dose was increased to $0.5 \mathrm{mg}$ twice per week.

\section{Discussion}

This case demonstrates some of the diagnostic difficulties associated with skull base tumors. Here, we reported a
Table 1 Prolactin levels and MRI findings during follow-up from diagnosis until postpartum.

\begin{tabular}{|c|c|c|}
\hline Time of measurement & $\begin{array}{c}\text { Prolactin levels } \\
\text { (ng/mL) (with dilution) }\end{array}$ & $\begin{array}{l}\text { MRI pituitary } \\
\text { lesion size }(\mathrm{cm})\end{array}$ \\
\hline \multicolumn{3}{|l|}{ Preoperative (baseline) } \\
\hline & $142.5 \mathrm{ng} / \mathrm{mL}^{*}$ & \\
\hline With dilution & 3380.95 & $6.2 \times 4.22$ \\
\hline Postoperative & 15.3 & $4.7 \times 2.9$ \\
\hline Intrapartum & 18.0 & $4.9 \times 3.4$ \\
\hline Postpartum & 16.6 & $5.0 \times 3.7$ \\
\hline
\end{tabular}

*Initial measurement, without dilution.

patient with an unusual clinical presentation, in which the diagnosis of an invasive, giant macroprolactinoma was only made following surgical debulking and the discovery of an elevated PRL level after retesting of the serum prolactin sample with serial dilutions. To the best of our knowledge, this is the second case of an invasive, giant macroprolactinoma during pregnancy. In contrast to the first case that was diagnosed during pregnancy and managed with bromocriptine, our patient was diagnosed before conception and was managed with $\mathrm{CAB}$ during the entire course of her pregnancy. PRL-secreting adenomas are the most common pituitary tumors in women of childbearing age (3). Prolactinomas are classified based on their size as macroadenomas $(>1 \mathrm{~cm})$ or microadenomas $(<1 \mathrm{~cm})(4,5)$. Approximately $90 \%$ of pituitary tumors are intrasellar adenomas, while the remainder are macroadenomas. Giant prolactinomas $(>4 \mathrm{~cm})$ are a rare pituitary tumor subtype associated with high serum PRL levels that are $>1000 \mathrm{ng} / \mathrm{mL}$ (5). The correct diagnosis of this subtype is critical, as surgical resection is associated with significant morbidity and mortality $(15,16)$; treatment with DA can effectively lower serum PRL levels and significantly reduce tumor size in most cases (17).

Pituitary adenomas are diverse, even in the case of symptomatic hyperprolactinemia. Although nonfunctioning adenomas can exhibit stalk effects and PRL levels can reach up to $200 \mathrm{ng} / \mathrm{mL}$, diagnostic challenges remain a primary concern in such scenarios $(18,19)$. The presence of a high-dose hook effect, which occurs when there is a lack of enough antibodies to bind to each end of all antigenic PRL peptides, can further complicate diagnosis. Given that extreme levels of PRL are present in the case of giant macroprolactinomas, most of PRL will form a single antibody; therefore, only a few PRL peptides will be detected using the most commonly used assays, resulting in falsely low serum PRL levels and subsequent misdiagnosis, ultimately leading to unnecessary pituitary surgery with serious morbidities. Presence of the hook 
effect can be avoided in monoclonal sandwich assays using two-step processing or by serial dilution in one-step processing $(20,21)$.

$\mathrm{CAB}$ is the first-line treatment for giant macroprolactinomas; extensive evidence has shown the effectiveness and safety of CAB. This DA can normalize PRL levels by around $69-75 \%$, and it can also achieve a 98\% decrease in PRL levels at doses of $3.5 \mathrm{mg}$ per week. $\mathrm{CAB}$ can further reduce tumor sizes anywhere from 26 to 90\% (22).

Once pregnancy occurs in women with prolactinomas, there are two main concerns during pregnancy; first, there is the increase in tumor size during pregnancy that results from increased estrogen levels, which induces lactotroph cell hyperplasia (23). Women with microadenomas have a $<3 \%$ chance of symptomatic tumor growth during pregnancy as compared to a $25-30 \%$ chance of tumor growth in women with recently diagnosed macroadenomas and/or without previous long-term treatment $(23,24)$. Second, there is the effect of DA on early fetal development and pregnancy outcomes. The data available on the use of bromocriptine in more than 6000 pregnancies (studied at the time of conception or during pregnancy) indicated no increased risk of spontaneous abortions, congenital malformations or neonatal abnormalities $(25,26)$. Similarly, CAB studies showed no significant difference in the incidence of spontaneous abortions, ectopic pregnancies or neonatal malformations when compared to the general population $(27,28)$.

The latest guidelines recommend discontinuing DA in women with microadenomas as soon as pregnancy is confirmed, as excellent outcomes can be achieved with close clinical monitoring. Conversely, women with large macroadenomas and those with extrasellar extensions need to continue DA therapy throughout the pregnancy, as the risk of tumor enlargement is relatively high (14). CAB is more efficacious and presents with better tolerability when it is used to reduce PRL levels and control tumor size when compared with bromocriptine; however, no studies have examined these outcomes during pregnancy as yet. In the case presented here, once pregnancy was confirmed, and considering the risk of tumor enlargement and the potential need for surgery, it was decided to continue lower-dose $\mathrm{CAB}$ treatment during pregnancy. The choice to continue $\mathrm{CAB}$ was very effective, as it facilitated the course of the pregnancy, maintained the tumor size in this patient and was associated with favorable neonatal outcomes.

To date, publications on the use of $\mathrm{CAB}$ during pregnancy have been limited. There are only 16 cases of pregnant women treated with $\mathrm{CAB}$ during the entire pregnancy, including our patient; in all cases, no abnormalities were noted in the infants, except one case had an intrauterine death at 34 weeks when the mother had severe preeclampsia (29).

Serial PRL measurements during pregnancy are not necessary due to the high variability of PRL concentrations during pregnancy (4). In this patient, given the initial tumor characteristics, PRL measurements were carried out throughout the pregnancy and always remained within the normal range.

MRI examinations during pregnancy are not required; however, they can be performed without gadolinium, as there is no evidence of harm to the fetus from either MRI scans or gadolinium (9). In this patient, and given the potential risk of tumor enlargement, an MRI scan of the pituitary was performed in the second trimester without gadolinium; it showed a mild increase in the size of the adenoma when compared with the previous MRI. Moreover, there are no data to suggest that breastfeeding is associated with a significant increase in serum PRL levels or in the risk of tumor growth $(27,30)$. Thus, women can breastfeed normally, but the patient in our case was not able to lactate as she was on DA.

Pregnancycaninduceremission of hyperprolactinemia, and macroprolactinomas can occur in $64-70 \%$ of cases $(31,30)$. The mechanism underlying this phenomenon is unclear, but it may be attributed to the auto-infarction of the tumor (32). In our patient, the last MRI scan that was performed 2 months after delivery showed moderate progression, primarily noted in the rightmiddle cranial fossa and at the roof of the nasopharynx (Fig. 2C and D). Data on postpartum remission rates for these tumors are very limited. Some studies reported significant reductions in PRL levels and tumor volume postpartum when compared with values obtained before pregnancy $(31,33)$. The impact of pregnancy on giant prolactinomas has not yet been reported. Interestingly, in our patient, treatment with CAB had to continue, as the patient did not demonstrate tumor shrinkage after pregnancy. Furthermore, if the patient continues to experience further tumor expansion despite DA therapy, the clinical team may need to consider medical therapy with temozolomide, radiotherapy or surgical debulking.

\section{Conclusion}

Pregnancy in a patient with an invasive, giant macroprolactinoma is rare and its treatment is challenging. This case demonstrates the effectiveness 
and safety of $\mathrm{CAB}$ therapy during pregnancy. Although the patient's adenoma size increased slightly during pregnancy, she had a successful pregnancy with favorable fetal outcomes. Our case further supports the importance of making an accurate diagnosis and initiating proper treatment before pregnancy. Also, a multidisciplinary team involving endocrinologists, neurosurgeons and obstetricians is required for safe outcomes for both the mother and newborn.

\section{Declaration of interest}

The authors declare that there is no conflict of interest that could be perceived as prejudicing the impartiality of the research reported.

\section{Funding}

This research did not receive any specific grant from any funding agency in the public, commercial or not-for-profit sector.

\section{Patient consent}

Written informed consent has been obtained from the patient for publication of the submitted article and accompanying images.

\section{Acknowledgments}

English-language editing of this manuscript was provided by Journal Prep Services.

\section{References}

1 Daly AF, Rixhon M, Adam C, Dempegioti A, Tichomirowa MA $\&$ Beckers A. High prevalence of pituitary adenomas: a crosssectional study in the province of Liege, Belgium. Journal of Clinical Endocrinology and Metabolism 200691 4769-4775. (https://doi. org/10.1210/ jc.2006-1668)

2 Fernandez A, Karavitaki N \& Wass JA. Prevalence of pituitary adenomas: a community-based, cross-sectional study in Banbury (Oxfordshire, UK). Clinical Endocrinology 201072 377-382. (https:// doi.org/10.1111/j.1365-2265.2009.03667.x)

3 Raappana A, Koivukangas J, Ebeling T \& Pirilä T. Incidence of pituitary adenomas in Northern Finland in 1992-2007. Journal of Clinical Endocrinology and Metabolism 201095 4268-4275. (https:// doi.org/10.1210/jc.2010-0537)

4 Almalki MH, Alzahrani S, Alshahrani F, Alsherbeni S, Almoharib O, Aljohani N \& Almagamsi A. Managing prolactinomas during pregnancy. Frontiers in Endocrinology 20156 85. (https://doi. org/10.3389/fendo.2015.00085)

5 Siddiqui A, Chew N \& Miszkiel K. Unusual orbital invasion by a giant prolactinoma. British Journal of Radiology $2008 \mathbf{8 1} 259-262$.

6 Buchfelder M, Fahlbusch R, Adams EF, Kiesewetter F \& Thierauf P. Proliferation parameters for pituitary adenomas. Acta Neurochirurgica 199665 18-21.

7 Scheithauer BW, Randall RV, Laws ER Jr, Kovacs KT, Howart E \& Whitaker MD. Prolactin cell carcinoma of the pituitary. Clinicopathologic, immunohistochemi- cal, and ultrastructural study of a case report with cranial and extracranial metastases. Cancer 1985 55 598-604.
8 Webster J, Piscitelli G, Polli A, Ferrari CI, Ismail I \& Scanlon MF. A comparison of cabergoline and bromocriptine in the treatment of hyperprolactinemic amenorrhea. Cabergoline Comparative Study Group. New England Journal of Medicine 1994331 904-909.

9 Biller BM, Molitch ME, Vance ML, Cannistaro KB, Davis KR, Simons JA, Schoenfelder JR \& Klibanski A. Treatment of prolactinsecreting macroadenomas with the once-weekly dopamine agonist cabergoline. Journal of Clinical Endocrinology and Metabolism $1996 \mathbf{8 1}$ 2338-2343.

10 Colao A, Di Sarno A, Sarnacchiaro F, Ferone D, Di Renzo GF, Merola B, Annunziato L \& Lombardi G. Prolactinomas resistant to standard dopamine agonists respond to chronic cabergoline treatment. Journal of Clinical Endocrinology and Metabolism 199782 876-883.

11 Gillam MP, Molitch ME, Lombardi G \& Colao A. Advances in the treatment of prolactinomas. Endocrine Review 200627 485-534.

12 Moraes AB, Silva CM, Vieira Neto L \& Gadelha MR. Giant prolactinomas: the therapeutic approach. Clinical Endocrinology 2013 79 447-456.

13 Konopka P, Raymond JP, Merceron RE \& Seneze J. Continuous administration of bromocriptine in the prevention of neurological complications in pregnant women with prolactinomas. American Journal of Obstetrics and Gynecology 1983146 935-938.

14 Melmed S, Casanueva FF, Hoffman AR, Kleinberg DL, Montori VM, Schlechte JA, Wass JA \& Endocrine Society. Diagnosis and treatment of hyperprolactinemia: an Endocrine Society clinical practice guideline. Journal of Clinical Endocrinology and Metabolism 201196 273-288.

15 Shahzad H, Sheikh A \& Sheikh L. Cabergoline therapy for macroprolactinoma during pregnancy: a case report. BMC Research Notes 20125606

16 Pia HW, Grote E \& Hildebrandt G. Giant pituitary adenomas. Neurosurgical Review 19858 207-220.

17 Acharya SV, Gopal RA, Menon PS, Bandgar TR \& Shah NS. Giant prolactinoma and effectiveness of medical management. Endocrine Practice 201016 42-46.

18 Klijn JG, Lamberts SW, de Jong FH, Docter R, Van Dongen KJ $\&$ Birkenhäger JC. The importance of pituitary tumour size in patients with hyperprolactinaemia in relation to hormonal variables and extrasellar extension of tumour. Clinical Endocrinology 198012 341-355. (https://doi.org/10.1111/j.1365-2265.1980. tb02719.x)

19 Smith MV \& Laws ER Jr. Magnetic resonance imaging measurements of pituitary stalk compression and deviation in patients with nonprolactin-secreting intrasellar and parasellar tumors: lack of correlation with serum prolactin levels. Neurosurgery 199434 834-839. (https://doi.org/10.1227/00006123-19940500000007)

20 Barkan A \& Chandler W. Giant pituitary prolactinoma with falsely low serum prolactin: the pitfall of the "high-dose hook effect": case report. Neurosurgery 199842 913-915.

21 Unnikrishnan A, Rajaratnam S, Seshadri M, Kanagasapabathy A \& Stephen D. The 'hook effect' on serum prolactin estimation in a patient with macroprolactinoma. Neurology India 200149 78-80.

22 Almalki MH, Buhary B, Alzahrani S, Alshahrani F, Alsherbeni S, Alhowsawi G \& Aljohani N. Giant prolactinomas: clinical manifestations and outcomes of 16 Arab cases. Pituitary 201518405 (https://doi.org/10.1007/s11102-014-0588-3)

23 Molitch ME. Prolactinoma in pregnancy. Best Practice and Research: Clinical Endocrinology and Metabolism 201125 885-896.

24 Hoffman AR, Melmed S \& Schlechte J. Patient guide to hyperprolactinemia diagnosis and treatment. Journal of Clinical Endocrinology and Metabolism 201196 35-36.

25 Krupp P \& Monka C. Bromocriptine in pregnancy: safety aspects. Klinische Wochenschrift 198765 823-827. (https://doi.org/10.1007/ BF01727477) 
26 Krupp P, Monka C \& Richter K. The safety aspects of infertility treatments. In Program of the Second World Congress of Gynecology and Obstetrics. Rio de Janeiro, Brazil, 1988.

27 Lebbe M, Hubinont C, Bernard P \& Maiter D. Outcome of 100 pregnancies initiated under treatment with cabergoline in hyperprolactinaemic women. Clinical Endocrinology $2010 \mathbf{7 3}$ 236-242.

28 Canfield MA, Honein MA \& Yuskiv N. National estimates and race/ ethnic-specific variation of selected birth defects in the United States, 1999-2001. Birth Defects Research Part A: Clinical and Molecular Teratology 200676 747-756. (https://doi.org/10.1002/ bdra.20294)

29 Glezer A \& Bronstein MD. Prolactinomas, cabergoline, and pregnancy. Endocrine 201447 64-69. (https://doi.org/10.1007/ s12020-014-0334-7)
30 Domingue ME, Devuyst F, Alexopoulou O, Corvilain B \& Maiter D. Outcome of prolactinoma after pregnancy and lactation: a study on 73 patients. Clinical Endocrinology $201480642-648$.

31 Auriemma RS, Perone Y, Di Sarno A, Grasso LF, Guerra E, Gasperi M, Pivonello R \& Colao A. Results of a single-center observational 10-year survey study on recurrence of hyperprolactinemia after pregnancy and lactation. Journal of Clinical Endocrinology and Metabolism 201398 372-379.

32 Molitch ME. Endocrinology in pregnancy: management of the pregnant patient with a prolactinoma. European Journal of Endocrinology 2015172 R205-R13. (https://doi.org/10.1530/EJE-14-0848)

33 Crosignani PG, Mattei AM, Severini V, Cavioni V, Maggioni P \& Testa G. Long-term effects of time, medical treatment and pregnancy in 176 hyperprolactinemic women. European Journal of Obstetrics and Gynecology and Reproductive Biology 199244 175-180.

Received in final form 13 July 2018

Accepted 31 July 2018 